\title{
National Institute of Health and Clinical Excellence Decision Processes Supported by UK High Court: The Debate about the Value of Alzheimer's Disease Drugs Continues
}

\author{
Peter J. Whitehouse \\ University Neurology Institute, Case Western Reserve University, Cleveland, OH, USA \\ E-mail: peter.whitehouse@case.edu
}

The assessment of the value of current and future drugs to treat dementia is a huge scientific, clinical, and social challenge, as pointed out by Ballard and colleagues [1]. Since the publication of the article, the controversies have not abated and will likely continue to grow as an international issue. The UK High Court ruled in favor of the National Institutes for Health and Clinical Excellence (NICE) in a case that challenged whether appropriate procedures were fairly carried out when the government agency recommended severely limiting access to cholinesterase inhibitors and memantine [2]. As the cost of drugs and concern about the industry's influence on medicine continues to grow, more governments are developing pharmacoeconomic programs modeled in part after NICE.

Several important lessons emerge from these discussions and debates. More high quality outcome studies would be desirable, but are expensive to undertake. Despite decade old efforts to encourage the pharmaceutical industry to take such studies of quality of life seriously, relatively little has been accomplished. Assessing the value of drugs in the elderly with dementia is clearly difficult. Although efficacy has been established in trials submitted for regulatory purposes, effectiveness in community practice has not been clearly established. The poor quality and evident bias in many post approval studies of the impact of the drugs actually undermines the industry's credibility. Moreover, nonpharmacological interventions may be better able to improve quality of lives, including those of the caregivers.

The biggest, mostly undiscussed issues are the price of drugs and the nature of their target. Why was the first drug (tacrine) priced at about four US dollars? Why have we not had more price competition in this industry which is said to be a model for global capitalism? Discussions about QUALYs (Quality Adjusted Life Years, i.e., length of life of a certain perceived utility) would be much different if the drugs cost four pennies a day. When the cholinesterase inhibitors start becoming generic in the next few years, the situation will change. We know the multinational pharmaceutical industry is in deep trouble in general. All the vaunted genomic medicine, combinatorial chemistry, high throughput screening, and improved trial approaches have not led to the much promised revolution in drugs for Alzheimer's disease. There are many reasons for this but the major one is that Alzheimer's disease is likely not a single condition and is intimately related to aging. Finding cost effective drugs is obviously very difficult. It may prove impossible, particularly in a world where other health concerns due to global warm- 
ing and the health of children rightfully increase in the years to come. Perhaps it is time to not only reassess the value of drugs, but the whole nature of our approach as individuals and societies to what we currently call Alzheimer's disease.

\section{References}

[1] C. Ballard, S. Sorensen and S. Sharp, Pharmacological Therapy for People with Alzheimer's Disease: The Balance of Clinical Effectiveness, Ethical Issues and Social and Healthcare Costs, J Alzheimers Dis 12 (2007), 53-59.

[2] R. Horton, NICE vindicated in UK's High Court, Lancet 370 (2007), 547-548. 